\title{
Neuropatía diabética y su asociación con síntomas ansiosos
}

\section{Diabetic Neuropathy and its Association with Anxiety Symptoms}

Recibido: 11 Diciembre 2020 | Aceptado: 09 Febrero 2021

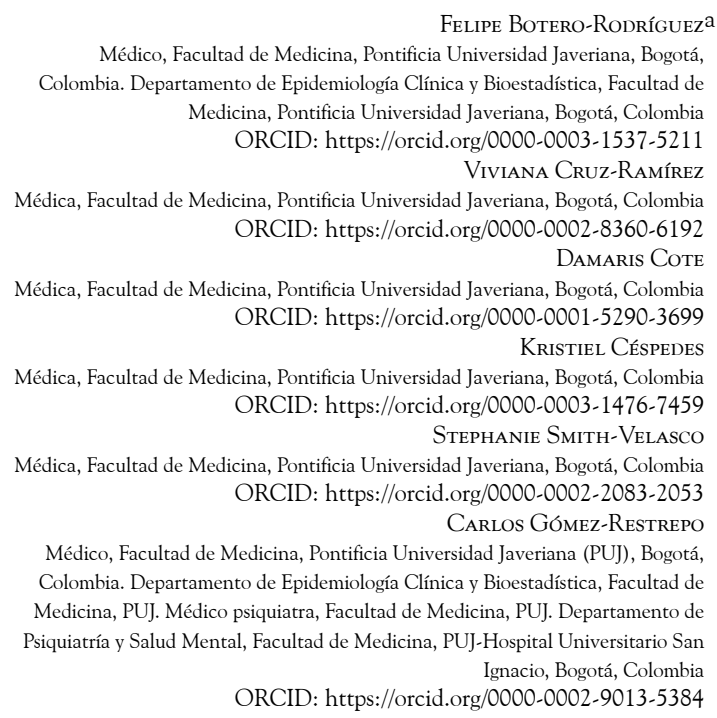

Felipe Botero-RodríGuez ${ }^{a}$

Médico, Facultad de Medicina, Pontificia Universidad Javeriana, Bogotá, Colombia. Departamento de Epidemiología Clínica y Bioestadística, Facultad de Medicina, Pontificia Universidad Javeriana, Bogotá, Colombia ORCID: https://orcid.org/0000-0003-1537-5211

Viviana Cruz-Ramírez

Médica, Facultad de Medicina, Pontificia Universidad Javeriana, Bogotá, Colombia ORCID: https://orcid.org/0000-0002-8360-6192 Damaris Cote

a Autor de correspondencia: felipe.botero@javeriana.edu.co

Cómo citar: Botero-Rodríguez F, Cruz-Ramírez V, Cote D, Céspedes K, Smith-Velasco S, Gómez-Restrepo C. Neuropatía diabética y su asociación con síntomas ansiosos. Univ. Med. 2021;62(2). https://doi.org/10.1 1144/Javeriana.umed62-2.neur

\section{RESUMEN}

Introducción: La neuropatía diabética es una complicación microvascular de la diabetes mellitus. Se caracteriza por una afectación en los nervios que produce un dolor tipo corrientazo, quemadura o punzada. Este dolor neuropático se ha asociado con alto riesgo de síntomas ansiosos en los pacientes que la padecen, lo que genera así una carga económica mayor en pacientes con neuropatía diabética. Objetivo: Describir la información actual en la literatura sobre la relación entre la neuropatía diabética sintomática y el riesgo de trastorno de ansiedad concomitante. Métodos: Búsqueda en paralelo por cuatro revisores en las bases de datos Ovid, Pubmed, SciELO y Cochrane. Resultados: De los 1140 artículos encontrados, 56 fueron evaluados en texto completo. Se encontró una prevalencia variada de síntomas ansiosos y depresivos en pacientes con neuropatía diabética, evaluados mediante diferentes cuestionarios y escalas. Algunos estudios encontraron asociación entre ansiedad, trastornos del sueño e inadecuado control glucémico. Se han reportado como factores de buen pronóstico la educación temprana y el manejo de dolor neuropático, siendo estos últimos los determinantes de la calidad de vida y la productividad en estos pacientes. Conclusión: La relación entre neuropatía diabética y los síntomas ansiosos puede ser bidireccional. Dados estos hallazgos y su posible impacto individual y en salud pública, se plantean oportunidades para llenar las brechas de prevención primaria, diagnóstico precoz y tratamiento en esta población, las cuales deberán definirse en futuras investigaciones.

Palabras clave

Neuropatía diabética; diabetes mellitus; ansiedad; salud pública. 


\begin{abstract}
Introduction: Diabetic neuropathy is a microvascular complication of diabetes mellitus. It is characterized by involvement in the nerves that produce a pain like rush, burn or stitch. This neuropathic pain has been associated with a high risk of anxiety in patients with it, thus generating a more significant economic burden in patients with symptomatic diabetic neuropathy. Aim: To describe current literature on the relationship between symptomatic diabetic Neuropathy and the risk of concomitant anxiety symptoms. Method: We carried out a similar search by four reviewers in the Ovid, Pubmed, SciELO, and Cochrane databases. Results: Of the 1140 articles found, 56 were evaluated in full text. A varied prevalence of anxious and depressive symptoms was found in patients with symptomatic diabetic neuropathy, evaluated using different questionnaires and scales. Some studies found an association between anxiety symptoms, sleep disorders, and inadequate glycemic control. Early education and the management of neuropathic pain have been reported as factors of good prognosis, the latter being the determinants of quality of life and productivity in patients with symptomatic diabetic neuropathy. Conclusion: The relationship between diabetic neuropathy and anxiety symptoms can be bidirectional. Given these findings and their possible impact on the individual and public health, opportunities arise to fill the gaps in primary prevention, early diagnosis, and treatment in this population, which should be defined in future research.
\end{abstract}

Keywords

diabetic neuropathy; diabetes mellitus; anxiety; public health.

\section{Introducción}

La alta morbilidad de la diabetes mellitus (DM) $(1,2,3)$ se traduce en altos costos que, así como la prevalencia de la enfermedad, tienden a incrementar en países de altos, medianos y bajos ingresos, a través del tiempo $(4,5,6,7)$. Para el 2040, la Federación Internacional de Diabetes prevé que 642 millones de individuos padecerán DM $(8,9,10)$, y para el 2045, 42,3 millones de individuos diagnosticados se encontrarán en la región de las Américas (9). Ello genera que la DM se considere un problema de salud pública en esta región $(11,12)$. Adicionalmente, este trastorno metabólico puede presentar complicaciones micro y macrovasculares, entre las cuales se destaca la neuropatía diabética (ND), que constituye una de las principales complicaciones de la DM $(13,14,15)$. Esta, a su vez, puede verse asociada con alto riesgo de síntomas ansiosos en los pacientes con DM.

La ND es una afectación de los nervios que resulta de la hiperglucemia, lo que produce un dolor caracterizado por ser de tipo corrientazo, quemadura o punzada, aunque también se puede presentar asintomáticamente. La ND afecta tantos sistemas y de tan diversas maneras que su clasificación es difícil y no existe una que sea mundialmente aceptada. No obstante, en términos generales, esta condición suele ser focal o multifocal, y afectar cierto tipo de fibra nerviosa (tipo C) o un sistema en particular (15).

Frente al dolor por la ND, se han descrito asociaciones con diversos trastornos mentales, tanto asociados como causados por esta. Existe evidencia de que cerca del $50 \%$ de los pacientes diabéticos presenta ND y de estos, aproximadamente, el $4 \%$ ha presentado depresión o ansiedad en su vida, sobre todo más en mujeres que en hombres $(5,1 \%$ vs. $3,4 \%)$. Otros estudios han indicado que el dolor crónico con características neuropáticas se presenta en uno de cada cinco pacientes diabéticos y que si no se controla adecuadamente, puede repercutir de manera negativa en la calidad de vida de los pacientes $(16,17)$.

Por otro lado, la presencia de trastornos mentales en pacientes con ND se ha asociado con un mayor riesgo de desarrollar trastornos del sueño o tener pobre control de otras comorbilidades (hipertensión arterial, obesidad y dislipidemia), además de incrementar per se los síntomas ansiosos (18). Pedras et al. (19) hallaron que en pacientes diabéticos con úlceras en sus miembros inferiores, remitidos para manejo ablativo, existía mayor riesgo de depresión y síntomas ansiosos, los cuales se exacerbaban durante el periodo previo a la cirugía. Adicionalmente, se ha descrito que los pacientes con ND y ansiedad tienen una mayor prevalencia de complicaciones relacionadas con la DM y comorbilidades, sobre todo enfermedad cerebrovascular, enfermedad arterial periférica y problemas dérmicas, lo que resulta en mayor morbimortalidad $(16,20)$.

Dicho lo anterior, y teniendo en cuenta que los síntomas de ansiedad son altamente prevalentes 
y generan una mayor carga de enfermedad (21, 22), sumado a la carga emocional que conlleva la ND, y que su adecuado tratamiento demanda modificaciones en varios aspectos de la vida que pueden desencadenar alteraciones en el estado físico y mental de la persona, se considera necesario detallar la asociación entre la ND con los síntomas ansiosos, así como mencionar la carga económica que esta implica y el potencial impacto sobre la calidad de vida de pacientes con estas comorbilidades.

\section{Métodos}

Se utilizaron diferentes bases de datos y motores de búsqueda: Ovid, Pubmed, SciELO y Cochrane. La búsqueda la llevaron a cabo, en paralelo, cuatro revisores con conocimientos del área de la salud, con el fin de extraer información de manera pareada. La búsqueda utilizó los siguientes términos indexados y libres: diabetic neuropathies, diabetic foot, dorsal root ganglion, diabetes complications, anxiety disorders, anxiety, diabetic angiopathy, diabetic microangiopathy, diabetic neuropathy, anxiety assessment, complicaciones diabéticas, neuropatías diabéticas, pie diabético, ganglio de la raíz dorsal, trastorno de ansiedad, ansiedad, angiopatía diabética, microangiopatía diabética, neuropatía diabética, manejo de ansiedad. La información se limitó a estudios llevados a cabo en humanos. Se incluyeron artículos en inglés, español y portugués.

Para seleccionar los artículos, estos debían mencionar la relación entre trastorno ansioso y DM, mencionar la carga económica que esta pudiese tener o incluir sus repercusiones en la calidad de vida de los pacientes. No hubo filtro por tipo de estudio, población o de medición utilizada para expresar la carga económica o la calidad de vida. Finalmente, se produjeron dimensiones que sintetizaran adecuadamente los hallazgos de la revisión y, así mismo, se describieron.

\section{Resultados}

Se encontraron 794 artículos publicados entre 2007 y 2019. Tras la eliminación de duplicados, eliminación de resúmenes de póster y filtro por título y resumen, se excluyeron 738 referencias. Finalmente, se evaluaron en texto completo los artículos restantes y se seleccionaron 21, a partir de los cuales se formalizaron las dimensiones descritas a continuación.

Aproximación fisiopatológica del trastorno de ansiedad en neuropatía diabética

El dolor neuropático es causado por múltiples etiologías, que producen diferentes manifestaciones y pueden variar según la localización. Esto implica que los tipos de fibras nerviosas afectadas también cambian según el tipo de afectación. No obstante, parece existir un mecanismo común entre la ND y los síntomas ansiosos. Este mecanismo inicia con el rol del péptido semejante al glucagón de tipo 1 (GLP-1) en el sistema nervioso, ya que este actúa como un neurotransmisor que reduce la inducción de apoptosis de neuronas hipocampales; pero también actúa disminuyendo los estímulos del hambre y puede estimular comportamientos ansiosos (23). Otra teoría incluye la falta de protección del tejido cerebral al estrés oxidativo, por medio del barrido de radicales libres, mecanismo que es llevado a cabo por diferentes vitaminas (por ejemplo, E, C y A). En relación con esto, Mazloom et al. (24) describieron una disminución de la ansiedad en pacientes que recibieron vitamina A durante seis semanas y, así, demostraron una asociación entre la alteración de concentraciones de vitamina A y el desarrollo de síntomas ansiosos.

Asimismo, Mazloom et al. (24) informaron que los estados de estrés como la ansiedad tienen un efecto neurotóxico a través de radicales reactivos que afectan la plasticidad sináptica y la morfología de las dendritas. Igualmente, evidenciaron una relación entre el estrés oxidativo celular y regulación de la ansiedad. Estos mecanismos se presentan 
de manera proporcional al progreso de la enfermedad, pues se ha documentado mayor magnitud en aquellos pacientes que presentan $\mathrm{ND}$, lo que explica esta asociación con una mayor prevalencia de síntomas ansiosos. No obstante, estudios con modelos animales proponen más mecanismos que relacionan las dos condiciones y señalan la necesidad de realizar investigaciones en humanos (25).

También se ha señalado un mecanismo inverso, en el que los síntomas de ansiedad y de estrés aumentan las concentraciones de glucosa en la sangre, lo que fomenta el desarrollo de la ND. En situaciones de estrés, el hipotálamo secreta la hormona liberadora de corticotropina, que estimula la glándula pituitaria para la liberación de adrenocorticotropina, y ello lleva a la secreción de glucocorticoides. Dentro de ellos, el cortisol estimula la gluconeogénesis, para proporcionar energía inmediata. Esto se ha constatado en múltiples estudios, que han evidenciado que un adecuado manejo de la ansiedad ha ayudado a mantener óptimas cantidades de glucosa en la sangre, lo cual favorece la percepción de enfermedad en estos pacientes y la adherencia al tratamiento (26).

\section{Asociación clínica entre neuropatía diabética y ansiedad}

Se ha descrito que en las personas diabéticas se presentan de dos a cuatro veces más síntomas psicológicos - $\mathrm{y}$, sobre todo, afectivos - que el resto de la población $(26,27)$. Específicamente, la ND, debido al dolor crónico, se ha relacionado con disfunción física, malestar emocional y disminución en la calidad de vida, humor y sueño; asimismo, se ha asociado con síntomas ansiosos $(13,16)$. Dicha asociación se describe con mayor frecuencia mujeres y en individuos que presentan otras comorbilidades, por ejemplo, pie de Charcot $(19,26)$ o trauma craneoencefálico (25).

Por otro lado, los síntomas de ansiedad, usualmente relacionados con estrés, han demostrado estar fuertemente vinculados con una conceptuación negativa de la DM, que va empeorando según la severidad de la diabetes (26). Esto cobra importancia, porque pueden obstaculizar el adecuado tratamiento de la DM y, por ende, de la ND. Algunos autores sugieren una evaluación integral del trastorno ansioso y síntomas mentales en pacientes que son diagnosticados con ND (27).

También se halló en la literatura que la minoría de pacientes recibe un adecuado tratamiento para la ND (16), a pesar de que diversos estudios han demostrado la disminución del dolor neuropático con el uso de antidepresivos, que también pueden llevar a la posterior reducción de los síntomas ansiosos (24). Al contrario, se ha encontrado como opción terapéutica la exenatida, análoga del GLP-1, que proporciona efectos ansiolíticos y antidepresivos, además de revertir la ND $(28,29)$. Asimismo, hay una correlación positiva entre las concentraciones de hemoglobina glucosilada y el puntaje de las escalas para ansiedad $(r=0,246 ; \mathrm{p}<0,05)(30$, 31).

Vale resaltar que una de las complicaciones importantes de la ND es el pie diabético. $\mathrm{Al}$ evaluar la relación entre pie diabético y salud mental, se ha documentado que aquellos pacientes con pie diabético presentaban puntajes más altos para síntomas ansiosos y depresivos de acuerdo con la Escala Hospitalaria de Ansiedad y Depresión (32). Respecto a las medidas de frecuencia, se ha estimado en esta población una prevalencia de síntomas ansiosos del 8,0 $\%$, depresivos del $24,7 \%$ y combinados del 5,4 \%. Se describió cómo los síntomas de ansiedad se asociaron significativa y positivamente con la presencia de dolor neuropático crónico (OR $=4,0 ;$ IC95 \%: 1,9-8,4; p < 0,0001). De igual forma, los síntomas de depresión se asociaron con dolor neuropático crónico (OR $=2,1$; IC95 \%: $1,4-3,3 ; p=0,001)$. Los síntomas combinados se relacionaron de manera significativa con dolor neuropático crónico (OR = 5,2; IC95 \%: $1,9-14,0 ; \mathrm{p}=0.001)$. Estos resultados sugieren que los pacientes con dolor neuropático tienen mayor oportunidad de presentar síntomas de ansiedad, depresión y ansiedad junto a depresión $(33,34)$. 
Otros factores asociados reconocidos fueron: sexo femenino, tener ingresos bajos, enfermedad crónica concomitante o pobre calidad de sueño (35). Con relación al sexo femenino como factor de riesgo, se encontró una mayor prevalencia de síntomas ansiosos en mujeres que en hombres $(17,6 \%$ vs. $10,0 \%$, respectivamente) $(31,36)$.

Carga de la enfermedad (gasto económico e impacto en la calidad de vida)

La diabetes y, sobre todo, sus complicaciones ocupan uno de los primeros lugares en la lista de carga de la enfermedad, en cuanto a las enfermedades no transmisibles en el ámbito global, y representan una carga económica significativa. Los cálculos de la Federación Internacional de Diabetes indican que en 2010 el gasto en diabetes representó un $9 \%$ del gasto sanitario en América del Sur y Central, y un $14 \%$ en América del Norte. Se estima un aumento por año de entre un $5 \%$ y un $7 \%$ en gasto sanitario secundario a DM y sus complicaciones $(37,38)$.

Con cierta frecuencia, los pacientes diabéticos presentan síntomas de ansiedad y depresión, usualmente en el periodo que abarca el diagnóstico, puesto que conlleva cambios de hábitos para el control de la enfermedad. Además, la ansiedad es una barrera importante en estos pacientes, ya que dificulta el inicio del tratamiento tanto del trastorno psiquiátrico como de la enfermedad metabólica, dado por conductas evitativas, disminución en la adherencia, entre otras (39).

En cuanto a la calidad de vida, Brod et al. (40) encontraron limitaciones significativas en diversos ámbitos y funciones de cada persona, por ejemplo, en funciones físicas (como la movilidad) o funciones diarias (como el aspecto laboral, el psicosocial, en el sueño, etc.). Con sus resultados señalaron que la ND, al afectar directamente la funcionalidad y el bienestar del paciente diabético, incrementa de forma considerable la carga de enfermedad y los síntomas ansiosos en los pacientes y sus familiares.

\section{Discusión}

En la revisión hecha para este artículo se planteó una asociación bidireccional entre la ND y los síntomas ansiosos. Aunque su mecanismo fisiopatológico no está del todo dilucidado, se han propuesto diversas teorías sobre su relación. Una de ellas es la acción de los elevados niveles de ansiedad sobre ejes hormonales que controlan el estrés, lo que genera así un mal control glucémico, que produce estrés oxidativo y una secundaria lesión neuropática $(31,41,42)$. En contraparte, un dolor crónico por ND implica un estado oxidativo constante y provoca malestar en el paciente, lo cual se correlaciona con un aumento de los niveles de ansiedad $(28,43,44)$. Vale la pena explorar los síntomas de la ND como posibles variables de confusión, en la medida en que el dolor se asocia tanto con ND como con síntomas depresivos, sin hacer parte de la vía causal o sin ser un eslabón intermedio. No obstante, no descartamos también una posible vía causal, en la que la ND produce dolor, y al no haber un adecuado control, genera síntomas ansiosos.

Adicionalmente, se encontraron asociaciones epidemiológicas entre las enfermedades mentales y las enfermedades físicas crónicas, tanto en niños como en adultos (45, 46, 47, 48, 49). Al investigar sobre este tema, se plantean oportunidades para acortar las brechas de prevención primaria, diagnóstico precoz y tratamiento en esta población, y se abre la posibilidad del desarrollo de futuras investigaciones e hipótesis de trabajo sobre esta asociación.

En relación con lo anterior, consideramos que la evaluación temprana sirve para crear estrategias de reducción de riesgo y para plantear objetivos de manejo que tengan una efectividad en el control de estas presentaciones concomitantes. Así mismo, con un manejo interdisciplinario se contribuye a reducir el estigma asociado con la búsqueda de servicios de salud mental, sumado a los beneficios del control sintomático mental y orgánico de esta población. Por lo tanto, se plantea que la integración de servicios de atención primaria y salud 
mental permitiría disminuir la carga económica relacionada con el manejo de enfermedades crónicas y trastornos mentales asociados $(13,45$, 46).

Estas oportunidades son importantes, al tener en cuenta que la expectativa de vida va en aumento (junto con los retos económicos y en salud que esto implica) $(50,51)$; por lo tanto, también aumenta la supervivencia de las personas con enfermedades crónicas (mentales y físicas). Por otro lado, es fundamental considerar la evaluación del estado mental para un diagnóstico y un tratamiento adecuados de estos pacientes, y un abordaje multidisciplinario a la población con enfermedades crónicas de base, ya que constituyen un problema de salud pública en el cual debemos enfocarnos para tener resultados óptimos en estas poblaciones vulnerables.

\section{Financiación y conflicto de intereses}

Los autores no declaran conflicto de intereses. Tampoco realizaron esta revisión con alguna fuente de financiación externa.

\section{Referencias}

1. Kong APS, Chan JCN. Hypoglycemia and comorbidities in type 2 diabetes. Curr Diab Rep. 2015 Oct;15(10):80. https://doi.org/10.1007 /s11892-015-0646-x

2. Burner TW, Rosenthal AK. Diabetes and rheumatic diseases. Curr Opin Rheumatol. 2009;21(1):50-4.

3. Wielgosz A, Dai S, Walsh P, McCreaLogie J, Celebican EM. Comorbid conditions in Canadians Hospitalized because of diabetes. Can J Diabetes. 2018 Feb;42(1):106-111. https://doi.or g/10.1016/j.jcjd.2017.03.004

4. Popkin BM, Kenan WR. Preventing type 2 diabetes: changing the food industry. Best Pract Res Clin Endocrinol Metab. 2016;30(3):373-83.
5. Popkin BM. Nutrition transition and the global diabetes epidemic. Curr Diab Rep. 2015;15(9):64. https://doi.org/10. 1007/s11892-015-0631-4

6. Zimmet P, Alberti KG, Magliano DJ, Bennett PH. Diabetes mellitus statistics on prevalence and mortality: facts and fallacies. Nat Rev Endocrinol. 2016;12(10):616-22.

7. Ruiz-Dionisio M, CastañoVásquez A, Rodríguez-Fernández R. Carga de enfermedad de diabetes mellitus en Bogotá, D. C., 2009-2015. Rev Investig Segur Soc Salud [internet]. 2018;20(2):44-55. Disponible en: http://app.saludcapital. gov.co/revistadigital/Paginas/VerArtic ulo.aspx

8. International Diabetes Federation. IDF diabetes atlas [internet]; 2015. Disponible en: https://idf.org/e-library/ epidemiology-research/diabetes-atlas.h tml

9. Comité de la Octava Edición de Diabetes Atlas. Diabetes atlas. Vol. 8. Federación Internacional de Diabetes; 2017.

10. International Diabetes Federation. IDF diabetes atlas. 6th ed.; 2013.

11. Aschner P, Aguilar-Salinas C, Aguirre L, Franco L, Gagliardino JJ, de Lapertosa SG, et al. Diabetes in South and Central America: an update. Diabetes Res Clin Pract. 2014;103(2):238-43.

12. Caporale JE, Calvo H, Gagliardino JJ. Costos de atención médica de personas con diabetes anteriores y posteriores a su hospitalización en Argentina. Rev Panam Salud Pública. 2006;20(6):361-8.

13. Boulanger L, Zhao Y, Foster TS, Fraser K, Bledsoe SL, Russell MW. Impact of comorbid depression or anxiety on patterns of treatment and economic outcomes among 
patients with diabetic peripheral neuropathic pain. Curr Med Res Opin. 2009;25(7):1763-73.

14. Backonja MM, Attal N, Baron R, Bouhassira D, Drangholt M, Dyck PJ, et al. Value of quantitative sensory testing in neurological and pain disorders: NeuPSIG consensus. Pain. 2013;154(9):1807-19.

15. Vinik AI, Nevoret M-L, Casellini C, Parson H. Diabetic neuropathy. Endocrinol Metab Clin North Am. 2013 Dec;42(4):747-87. https://doi.org /10.1016/j.ecl.2013.06.001

16. Walk D, Poliak-Tunis M. Chronic pain management: an overview of taxonomy, conditions commonly encountered, and assessment. Med Clin North Am. 2016;100(1):1-16.

17. Aaron RV, Fisher EA, De La Vega R, Lumley MA, Palermo TM. Alexithymia in individuals with chronic pain and its relation to pain intensity, physical interference, depression, and anxiety: a systematic review and meta-analysis. Pain. 2019;160(5):994-1006.

18. Chapman Z, Shuttleworth CMJ, Huber JW. High levels of anxiety and depression in diabetic patients with Charcot foot. J Foot Ankle Res. 2014;7:22.

19. Pedras S, Carvalho R, Pereira MG. A predictive model of anxiety and depression symptoms after a lower limb amputation. Disabil Health J. 2018 Jan;11(1):79-85. https://doi.org/10.101 6/j.dhjo.2017.03.013

20. Ahmad A, Abujbara M, Jaddou $\mathrm{H}$, Younes NA, Ajlouni K. Anxiety and depression among adult patients with diabetic foot: prevalence and associated factors. J Clin Med Res. 2018 May;10(5):411-418. https://doi.o $\mathrm{rg} / 10.14740 /$ jocmr $3352 \mathrm{w}$
21. Hartford JT, Endicott J, Kornstein SG, Allgulander C, Wohlreich MM, Russell JM, et al. Implications of pain in generalized anxiety disorder: efficacy of duloxetine. Prim Care Companion J Clin Psychiatry. 2008;10(3):197-204.

22. Burke JP, Sánchez RJ, Joshi A V, Cappelleri JC, Kulakodlu M, Halpern $\mathrm{R}$. Health care costs in patients with painful diabetic peripheral neuropathy prescribed pregabalin or duloxetine. Pain Pract. 2012;12(3):209-18.

23. Anderberg RH, Richard JE, Hansson C, Nissbrandt $H$, Bergquist F, Skibicka KP. GLP-1 is both anxiogenic and antidepressant; divergent effects of acute and chronic GLP-1 on emotionality. Psychoneuroendocrinology.

2016;65:54-66.

24. Mazloom Z, Ekramzadeh M, Hejazi $\mathrm{N}$. Efficacy of supplementary vitamins $\mathrm{C}$ and $\mathrm{E}$ on anxiety, depression and stress in type 2 diabetic patients: a randomized, single-blind, placebocontrolled trial. Pak J Biol Sci. 2013;16(22):1597-600.

25. Abou-El-Hassan H, Dia B, Choucair K, Eid SA, Najdi F, Baki L, et al. Traumatic brain injury, diabetic neuropathy and altered-psychiatric health: The fateful triangle. Med Hypotheses. 2017 Oct;108:69-80.

26. Pedras S, Carvalho R, Pereira MG. Predictors of quality of life in patients with diabetic foot ulcer: The role of anxiety, depression, and functionality. J Health Psychol. 2018 Sep;23(11):1488-1498. https://doi.org/ 10.1177/1359105316656769

27. McDade-Montez EA, Watson D. Examining the potential influence of diabetes on depression and anxiety symptoms via multiple sample confirmatory factor analysis. Ann Behav Med. 2011;42(3):341-51. 
28. Al-Maskari M, Al-Shukaili A, Al-Mammari A. Pro-inflammatory cytokines in Omani type 2 diabetic patients presenting anxiety and depression. Iran J Immunol. 2010;7(2):124-9.

29. Tarkun I. Exenatide treatment exerts anxiolytic- and antidepressantlike effects and reverses neuropathy in a mouse model of type- 2 diabetes. Med Sci Monit Basic Res. 2014;20:112-7.

30. Demir AB, Kotan Z, Kotan VA, Bora I, Ozkaya G. Pain, daytime sleepiness, anxiety and depression levels of patients with chronic neuropathic pain syndromes. J Neurol Sci. 2016;33(1):89-101.

31. Whitworth SR, Bruce DG, Starkstein SE, Davis WA, Davis TME, Bucks RS. Lifetime depression and anxiety increase prevalent psychological symptoms and worsen glycemic control in type 2 diabetes: The Fremantle Diabetes Study Phase II. Diabetes Res Clin Pract. 2016;122:190-7.

32. Ducat L, Rubenstein A, Philipson LH, Anderson BJ. A review of the mental health issues of diabetes conference. Diabetes Care. 2015;38(2):333-8.

33. Ghasemi M, Phillips C, Trillo L, De Miguel Z, Das D, Salehi A. The role of NMDA receptors in the pathophysiology and treatment of mood disorders. Neurosci Biobehav Rev. 2014 Nov;47:336-58. https://doi.o $\mathrm{rg} / 10.1016 / \mathrm{j}$.neubiorev.2014.08.017

34. Gil-Bona J, Sabaté A, Miguelena Bovadilla JM, Adroer R, Koo M, Jaurrieta E. Valor de los índices de Charlson y la escala de riesgo quirúrgico en el análisis de la mortalidad operatoria. Cir Esp. 2010;88(3):174-9.
35. Sun N, Lou P, Shang Y, Zhang P, Wang J, Chang G, et al. Prevalence and determinants of depressive and anxiety symptoms in adults with type 2 diabetes in China: a cross-sectional study. BMJ Open. 2016;6(8):e012540.

36. Rajput R, Gehlawat P, Gehlan D, Gupta R, Rajput M. Prevalence and predictors of depression and anxiety in patients of diabetes mellitus in a tertiary care center. Indian J Endocrinol Metab. 2016;20(6):746-51.

37. De Wit L, Jelsma JGM, van Poppel MNM, Bogaerts A, Simmons D, Desoye G, et al. Physical activity, depressed mood and pregnancy worries in European obese pregnant women: results from the DALI study. BMC Pregnancy Childbirth. 2015;15:158.

38. Organización Panamericana de la Salud (OPS). La carga económica de las enfermedades no transmisibles en la región de las américas: únete en la lucha contra las ENTs [internet]. 2012;4. Disponible en: http://www.paho.org/hq/index.php ?option $=$ com_docman\&task $=$ doc_vi ew\&gid $=161 \overline{5} 8$ \& Itemi

39. Antúnez M, Bettiol Menegaldo AA. Depresión en pacientes con diabetes tipo 2 que acuden a una consulta externa de medicina interna. Acta Médica Colomb. 2016;41(2):102-10. https://doi.org/10. 36104/amc.2016.579

40. Brod M, Pohlman B, Blum SI, Ramasamy A, Carson R. burden of illness of diabetic peripheral neuropathic pain: a qualitative study. Patient. 2015;8(4):339-48.

41. Vukojević Z, Pekmezović T, Nikolić A, Perić S, Basta I, Marjanović I, Lavrnić D. Correlation of clinical and neurophysiological findings with health-related quality of life in patients with diabetic polyneuropathy. Vojnosanit Pregl. 2014;71(9):833-8. 
42. Vileikyte L, Peyrot M, Gonzalez JS, Rubin RR, Garrow AP, Stickings D, et al. Predictors of depressive symptoms in persons with diabetic peripheral neuropathy: a longitudinal study. Diabetologia. 2009;52 (7):1265-73.

43. Bouwman V, Adriaanse MC, van 't Riet E, Snoek FJ, Dekker JM, Nijpels G. Depression, anxiety and glucose metabolism in the general dutch population: the new Hoorn study. PLoS One. 2010;5(4):e9971.

44. Dutheil S, Ota KT, Wohleb ES, Rasmussen K, Duman RS. High-fat diet induced anxiety and anhedonia: impact on brain homeostasis and inflammation. Neuropsychopharmacology. 2016;41(7):1874-87.

45. Scott KM, Von Korff M, Alonso J, Angermeyer M, Bromet EJ, Bruffaerts $\mathrm{R}$, et al. Age patterns in the prevalence of DSM-IV depressive/ anxiety disorders with and without physical co-morbidity. Psychol Med. 2008;38(11):1659-69.

46. Ferro MA. Major depressive disorder, suicidal behaviour, bipolar disorder, and generalised anxiety disorder among emerging adults with and without chronic health conditions. Epidemiol Psychiatr Sci. 2016;25(5):462-74.

47. Halfon N, Newacheck PW. Evolving notions of childhood chronic illness. JAMA. 2010;303(7):665-6.

48. Gómez-Restrepo C, Ramírez S, Tamayo Martínez N, Rodríguez MN, Rodríguez A, Rengifo H. Prevalencia de posibles trastornos mentales en niños con condiciones crónicas: resultado de la Encuesta Nacional de Salud Mental Colombia 2015. Rev Colomb Psiquiatr. 2016;45:135-40. htt ps://doi.org/10.1016/j.rcp.2016.08.005
49. Martínez NT, Gómez-Restrepo C, Ramírez S, Rodríguez MN. Prevalencia de trastornos del afecto y de ansiedad en personas con condiciones crónicas: resultado de la Encuesta Nacional de Salud Mental Colombia 2015. Rev Colomb Psiquiatr. 2016 Dec 1;45:141-6. https://doi.org/10.1016/j.rc p.2016.06.001

50. Cano Gutiérrez CA. Editorial. Univ Médica. 2019;60(4):1-2.

51. Villar L, Flórez CE, Forero D, Valencia-López N, Puerta N, Botero F. Protección económica a la población mayor en Colombia. En: Misión Colombia envejece: cifras, retos y recomendaciones. Bogotá: Fundación Saldarriaga Concha; 2015. p. 80-161. 